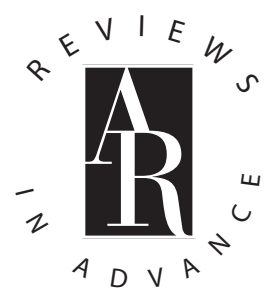

\title{
Physical Models of Plant Development
}

\section{Olivier Ali, ${ }^{1,2}$ Vincent Mirabet, ${ }^{2}$ Christophe Godin, ${ }^{1}$ and Jan Traas $^{2}$}

${ }^{1}$ Virtual Plants INRIA Team, UMR AGAP, 34398 Montpellier, France;

${ }^{2}$ Laboratoire de Reproduction et Développement des Plantes, Université de Lyon, INRA, CNRS, UCBL, Ecole Normale Supérieure-Lyon, 69364 Lyon Cedex 7, France; email: Olivier.Ali@ens-lyon.fr, Vincent.Mirabet@ens-lyon.fr, Christophe.Godin@inria.fr, Jan.Traas@ens-lyon.fr

Annu. Rev. Cell Dev. Biol. 2014. 30:2.1-2.20

The Annual Review of Cell and Developmental Biology is online at cellbio.annualreviews.org

This article's doi:

10.1146/annurev-cellbio-101512-122410

Copyright (c) 2014 by Annual Reviews. All rights reserved

\section{Keywords}

growth, simulation, mechanics

\section{Abstract}

The definition of shape in multicellular organisms is a major issue of developmental biology. It is well established that morphogenesis relies on genetic regulation. However, cells, tissues, and organism behaviors are also bound by the laws of physics, which limit the range of possible deformations organisms can undergo but also define what organisms must do to achieve specific shapes. Besides experiments, theoretical models and numerical simulations of growing tissues are powerful tools to investigate the link between genetic regulation and mechanics. Here, we provide an overview of the main mechanical models of plant morphogenesis developed so far, from subcellular scales to whole tissues. The common concepts and discrepancies between the various models are discussed. 


\section{Contents

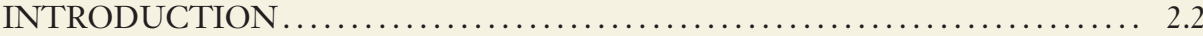

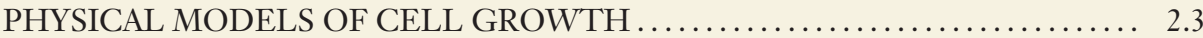

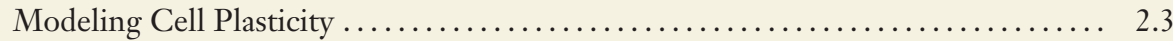 \\ Modeling the Growing Cell Wall................................... 2.5

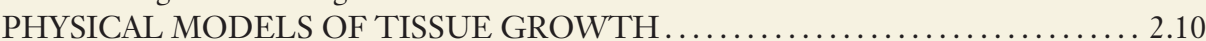 \\ A Conceptual Framework for Modeling Tissue Growth .................. 2.10 \\ Deterministic Models of Multicellular Tissues ......................... 2.12 \\ Integrating Mechanical Feedback in Multicellular Tissues ................. 2.15 \\ Toward the Integration of Stochasticity in Models of Multicellular Tissues ....... 2.16

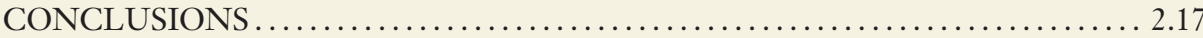

\section{INTRODUCTION}

The control of size and shape during the development of multicellular organisms is a major issue in developmental biology. Although many components of the molecular regulatory networks that coordinate development have been identified, genetic activities are often still expressed in relatively abstract and qualitative terms. As a result, it remains largely unclear how molecular action leads to specific changes in the geometry of growing tissues and organs.

The interaction between genes and shape is not direct. Indeed, to control changes in geometry, the molecular networks must interfere somehow with the physical structural elements of cells and tissues. Although it has long been recognized that morphogenesis relies on physical processes in which forces and mechanical properties play an important role (Boudaoud 2010, Lecuit \& Lenne 2007), until recently relatively little attention has been given to this aspect. This is partially because of difficulties in accessing physical parameters concerning local mechanics of cells and organs. Recent technical and conceptual advances have made it possible to incorporate biophysical approaches in the analysis of development, leading to renewed interest (Burgert 2006). Another bottleneck lies in the complexity of the data. Measurements of physical properties must be combined with protein-protein interactions, dynamic gene expression patterns, or complex tissue geometries. These complex data sets are impossible to analyze using simple visual inspection and intuitive, qualitative interpretations. Therefore, computational modeling is playing an increasingly important role in biology and has become crucial for the interpretation of the available data (Geitmann \& Ortega 2009). In particular, there is a need for models that integrate not only biochemical and geometrical aspects but also physical properties.

Physical models are already widely used in engineering to model the distribution of forces in various structures and shapes. In general, this involves a geometrical description of the structure and its external load, as well as hypotheses on mechanical properties of the material. Computational modeling is then used to calculate the resulting distribution of forces and stresses everywhere in the structure. This classical approach has been extremely successful and makes it possible to describe accurately the internal mechanical state of very complex structures. Producing physical models of living organisms is particularly challenging. This is not only because they contain many components but also because exchanges of mass and energy with the environment are prevailing, and the mechanical properties and shape of the structure itself are constantly changing.

Here, we focus on biophysical modeling approaches that are designed to capture morphogenesis in plants. Other processes, including rapid movements of plants (e.g., chiral opening of seed pods, 
rapid leaf movement), are not considered. Because we are reasoning within the context of complex systems with feedbacks at multiple scales, we discuss models that have been designed to address questions at both specific levels of organization and multiple scales, from molecular assemblies to organs. Starting from models and concepts that capture the physical basis of growth at the cellular level, we subsequently discuss how this translates to the tissue level, where multiple cells physically and biochemically interact.

\section{PHYSICAL MODELS OF CELL GROWTH}

Plant cells are under high internal turgor pressure and are prevented from bursting by the cell wall, a rigid shell composed of polysaccharides and proteins (Wolf et al. 2012). Because of this exoskeleton, plant cells within a tissue cannot move relative to each other. As a result, the global deformation of a tissue during its development is the cumulated effect of all the deformations undergone by each of its cells. Understanding the ground rules of cellular growth is therefore an essential step toward understanding shape development of plant organs.

\section{Modeling Cell Plasticity}

In 1965, Lockhart (1965) proposed a rheological model for plant cell growth in which the plant cell was reduced to its most fundamental elements: a cell wall and a cytoplasm. In this model, the cell wall was physically represented as a viscous shell filled with cytoplasm, considered as a pressurized, incompressible fluid (see Figure 1). The global size (length) of this system is dictated by the mechanical equilibrium between pressure-induced stresses and counterstresses exerted by the stretched wall. Modulation of this equilibrium, by changes in either osmolarity of the inner fluid or mechanical properties of the shell, can lead to irreversible (plastic) deformation and thus to cell growth.

This conceptual and powerful model of plant cell growth embodies the very core of most of the physical plant cell growth models to date. Its mathematical expression is given in Equation 1, where a change in length $l$ is related to the inner pressure $P$ of the cell:

$$
\begin{aligned}
\frac{1}{l} \frac{d l}{d t} & =m\left(P-P_{y}\right) & & \text { if } P>P_{y} \\
& =0 & & \text { if } P<P_{y}
\end{aligned},
$$

where $P_{y}$ is a threshold value above which growth is triggered (there is no growth if $P<P_{y}$ ) and $m$ is the extensibility of the cell wall. This variable accounts for the velocity of the wall growth once the pressure exceeds the threshold $P_{y}$ : The higher the $m$, the faster the growth under a given pressure.

This equation links quantifiable variables together (pressure and cell length), enabling experimental validation of Lockhart's theory (Green 1968, Green et al. 1971, Lockhart 1967). However, its simplicity leads to several limitations. The original Lockhart's equation describes irreversible cell wall expansion underlying growth at mechanical steady state, i.e., when mechanical stresses do not vary over time. However, the cell wall also shows elastic properties; in other words, it can undergo a reversible deformation when loaded (e.g., Cosgrove 1993, Schopfer 2006; for more recent experimental evidence, see Kierzkowski et al. 2012). To take this into account, Ortega (1985) extended Lockhart's model by introducing an elastic term, function of the cell wall's Young's modulus, noted $E$. This modulus quantifies the cell wall's stiffness; the higher its value, the stiffer the wall:

$$
\begin{aligned}
\frac{1}{l} \frac{d l}{d t} & =m\left(P-P_{y}\right)+\frac{1}{E} \frac{d P}{d t} & & \text { if } P>P_{y} \\
& =\frac{1}{E} \frac{d P}{d t} & & \text { if } P<P_{y}
\end{aligned} .
$$

Complex system: a system made of many interacting elements; exhibits emerging global properties not directly predictable from the properties of the individual components

Rheology: the study of flow of complex matters without explicitly taking into account their complex microscopic organizations 


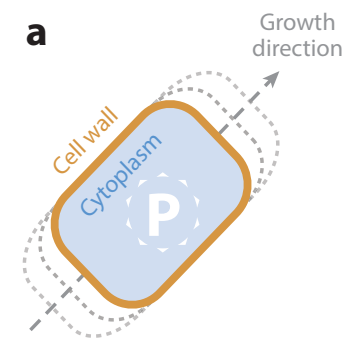

b
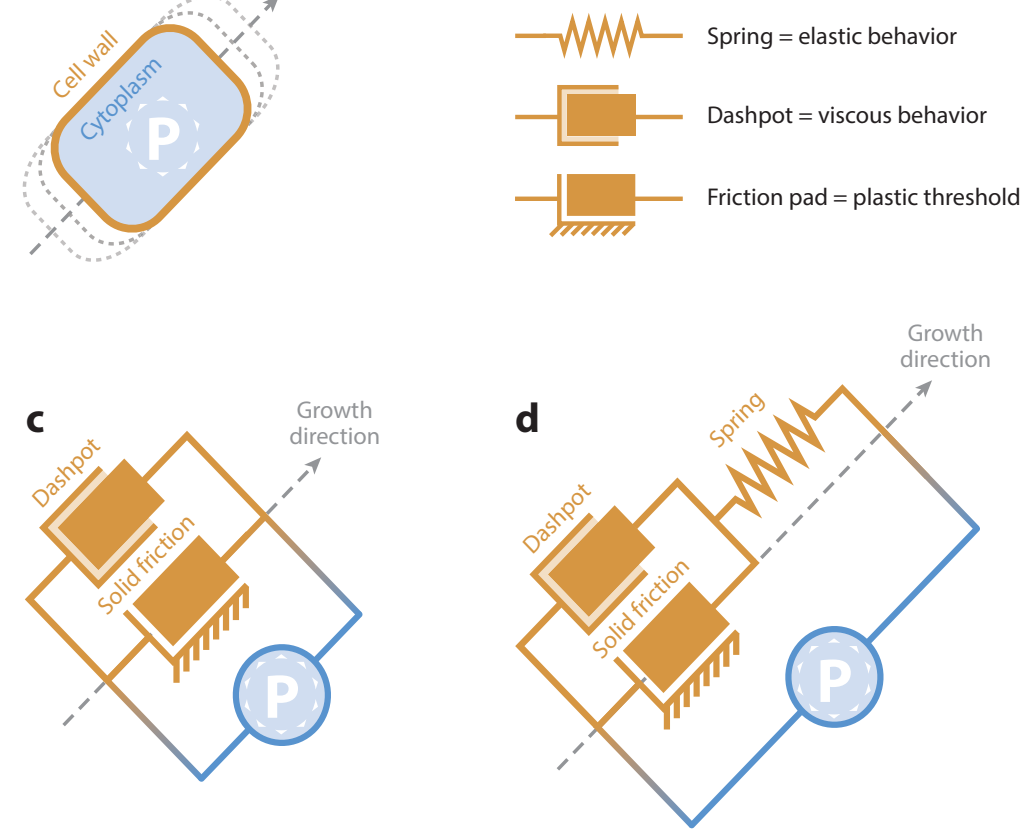

Figure 1

Schematic representation of a growing plant cell as captured by Lockhart's and Ortega's models: $(a)$ sketch of the considered biological actors, the cell wall and the cytoplasm. (b) Analogy between physical concepts and their schematic representations (see sidebar, Elasticity, Viscosity, and Plasticity, for further details). (c) Visual representation of Lockhart's model - first line of Equation 1. $(d)$ Visual representation of Ortega's model-first line of Equation 2.

The new elastic term brings a non-null contribution to the original Lockhart's equation only if the pressure changes over time. If $P$ is below the yielding value $P_{y}$, the change in length of the cell is due only to the elastic elongation of the wall and follows the evolution of the turgor pressure. This behavior is reversible: If $P$ increases, the cell expands; if it decreases, the cell shrinks. In this regime, the cell can be assimilated to a spring (see Figure 1). If $P$ is above the yielding value $P_{y}$, then new mechanisms, further detailed below, come into play, resulting in an additional plastic deformation, as described by Lockhart. The effective increase in size therefore results from the superposition of both elastic and plastic elongations. In this case, the cell can be assimilated to a spring and a dashpot connected in series (Figure 1), the latter accounting for the irreversible behavior of the cell wall.

Further extensions of Lockhart's model included the introduction of feedbacks. For instance, Green et al. (1971) observed changes in the yielding threshold upon changes in turgor pressure in the alga Nitella. To account for the suggested feedback, they coupled Equation 1 to an equation describing the dependency of the yielding threshold on the relative elongation rate. This led to the following system of equations:

$$
\left\{\begin{array}{l}
\frac{1}{l} \frac{d l}{d t}=m\left(P-P_{y}\right) \quad \text { if } P>P_{y} \\
\frac{d P_{y}}{d t}=b \frac{1}{l} \frac{d l}{d t}-s
\end{array} .\right.
$$




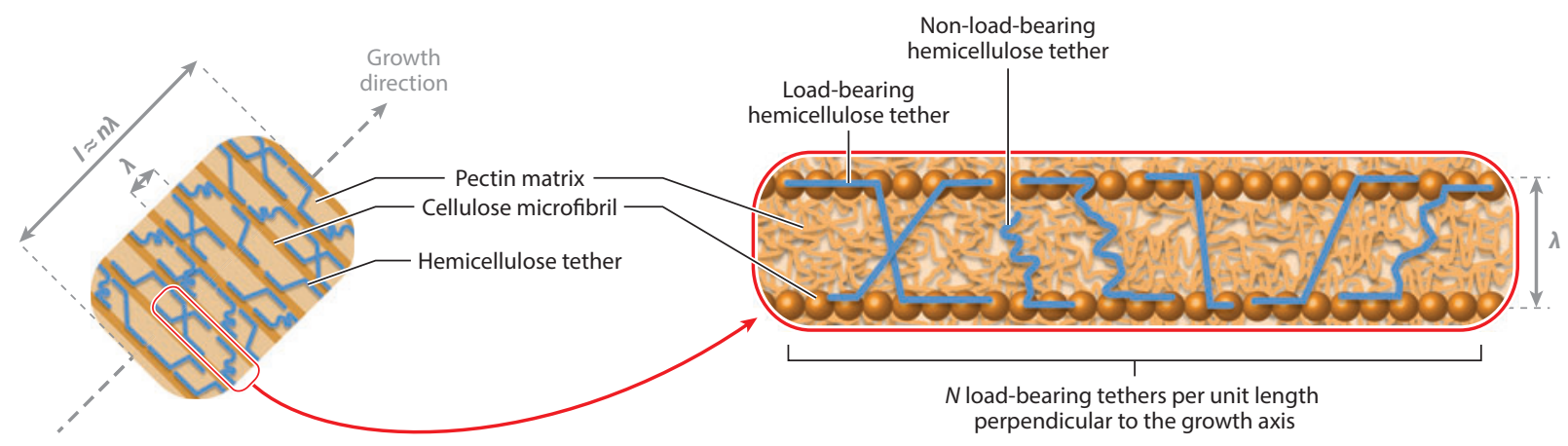

Figure 2

Representation of the cell wall as modeled by Passouria \& Fry (1992). $n=l / \lambda$ is the cellulose microfibril (CMF) linear density in the growth direction and $N$ is the hemicellulose linear density in the perpendicular direction. Adapted from Passioura \& Fry (1992).

In Equation 3b, the parameter $b$ describes the increase of the yielding threshold with growth. It thus leads to strain stiffening: The faster the cell grows, the higher the yielding threshold. The second term, $s$ (with $s>0$ ), decreases the yielding threshold, attesting for simple wall loosening.

These add-ons for elastic behavior and mechanical feedback to Lockhart's model show that extension of the initial concept is possible. This is achieved, however, at the price of increasing complexity and introducing variables that are potentially much more difficult to infer from measurements, such as $b$ or $s$. Another important variable that is not taken into account is the direction of growth (Baskin 2005). The Lockhart model describes expansion in one dimension and does not explain how growth directions are controlled.

\section{Modeling the Growing Cell Wall}

To circumvent the limitations of the initial Lockhart equation and to understand how properties like elasticity, mechanical feedbacks, and growth directions emerge and are regulated, a range of studies have used models that consider the underlying molecular complexity of the cell wall. The primary cell wall, which characterizes growing cells, is composed of entangled polymer networks constantly reshaping themselves and is made up of two major subnetworks (see Figure 2; for reviews, see Cosgrove 2005, Hamant \& Traas 2009, Peaucelle et al. 2012, Wolf et al. 2012):

- The pectin network, an amorphous matrix, is formed by pectin chains cross-linked through calcium-mediated bounds (Willats et al. 2006). Pectins are small polysaccharides produced within the cell and exocytosed in a methyl-esterified form into the existing cell wall. In their methyl-esterified form, pectin monomers can in principle not cross-link to each other. Once enzymatically demethyl-esterified, and in the presence of divalent cations (usually calcium), pectin monomers can cross-link through the formation of multiple chelation bonds. Note that demethyl-esterification can also render the pectin molecules more accessible for degrading enzymes. The formed network is dense and thought to be mainly isotropic. Depending on the species and tissue, the amount of pectin can vary between $2-30 \%$ of the wall.

- The cellulose network is formed by long cellulose microfibrils (CMFs) linked together by shorter hemicellulose chains. CMFs are long, filamentous structures, directly polymerized at the inner face of the wall by transmembrane cellulose synthase complexes. CMFs represent in many cases $30 \%$ or more of the cell wall. Because they can be micrometers long and occur in bundles that can be even longer, CMFs are usually seen as the major load-bearing structures in the wall. Several proteins have been identified that modify the wall structure

Strain:

a relative measure of deformation; defined, in $1 \mathrm{D}$, as the ration of the length variation over the initial length: $\varepsilon=\left(l-l_{0}\right) / l_{0}=\Delta l / l_{0}$ 
Deterministic system: a system in which the evolution along time is determined only by its initial conditions

Stochastic system: a system with at least one randomly fluctuating variable; consequently, its evolution is not totally determined by its initial conditions and make or break bonds between polysaccharide chains (Cho \& Cosgrove 2000, Fleming et al. 1997, McQueen-Mason et al. 1992). Note that CMF organization and orientation can vary substantially from cell to cell, from highly anisotropic to highly isotropic arrangements that have a profound influence on growth directions (Baskin 2005). The orientations of the fibrils are dependent on the microtubular, membrane-attached cytoskeleton. Microtubules are themselves often organized into highly ordered arrays, through mechanisms that are still poorly understood. There is strong evidence that microtubules directly guide the cellulose synthase complexes in the plasma membrane (Paredez et al. 2006). Therefore, microtubule orientations likely reflect the orientation of the latest deposited CMFs.

Modeling the cell wall at short timescales. The knowledge of wall composition summarized above has been used to design physical models of the growing cell wall. Some of them consider the wall as a closed system, i.e., a system with a fixed composition, describing in principle short-term behaviors, from a few seconds to hours.

Passioura \& Fry (1992) considered the simple case of a rapidly growing cell in which the $\mathrm{CMFs}$ are all aligned parallel to each other, perpendicular to the main axis of expansion and linked together by hemicellulose tethers of different lengths. Although the wall composition was supposedly constant, the hemicellulose cross-links between the elements could be broken, and new ones could be created. Based on this molecular description, the wall extensibility and threshold of Lockhart's equation emerge as a function of the density of load-bearing tethers between two adjacent CMFs, perpendicular to the growth axis (see Figure 2). The authors also provided a more mechanistic, molecular description of the strain-stiffening and wall-softening mechanisms mentioned above. As the cell elongates, cellulose fibrils are pulled apart, and consequently, an increasing number of the tethers become load bearing, which is the essence of strain stiffening. Wall loosening can be represented simply by introducing a term for the enzymatic-controlled cutting or unbinding rate of the hemicellulose. This can be translated into the following equations, where extensibility $m$ and global yielding threshold $P_{y}$ are functions of the total cell length $l$; time $t$; the tethers' cutting/unbinding rate $c$; and $b$, the number of tethers between two adjacent CMFs per unit length along the cell's growing axis (see Figure 2):

$$
\left\{\begin{array}{l}
m=\frac{e}{(b l-c t)} \\
{[2 p t] P_{y}=(b l-c t) p_{y} .}
\end{array}\right.
$$

Note that Equation $4 \mathrm{~b}$ is a time-integrated version of Equation $3 \mathrm{~b}$, also deduced from this molecular modeling. Constants $e$ and $p_{y}$ represent the extensibility and the yielding threshold of a single tether molecule, respectively. Veytsman \& Cosgrove (1998) went even further, studying the contribution of interpenetrated hydrogen-bonded networks of cellulose and glucans. Their model also converged toward a Lockhart-like behavior where the yielding threshold depends on the concentration of active glucan and cellulose sites and not on the strength of hydrogen bonds between them.

Both studies mentioned above explored deterministic models of walls structure, with the aim of understanding wall expansion in a qualitative manner. Another axis of investigation of the wall mechanical properties is through stochastic numerical simulations of the behavior of large numbers of interacting wall components. These simulations permit, for example, precise predictions of the effects of variations in the nanostructure on the local elastic moduli of the entire wall (Kha et al. 2010, Yi \& Puri 2012). These models are much more demanding in terms of computational power, with multiple equations describing the behavior of hundreds of molecules that must be solved simultaneously. Consequently, the typical size of the simulated wall never exceeds a few hundreds of square micrometers, and growth behavior is not taken into consideration. Nonetheless, these 
simulations provide accurate estimations of various mechanical characteristics of the cell wall that can be plugged into growth-oriented deterministic models.

Mechanical models at longer timescales, including wall synthesis. Because many biological events related to growth feature long time dynamics, models valid at long timescales have been developed as well. In these models, where wall synthesis is explicitly taken into account, the cell wall is seen as an open system, i.e., a system to which components can be added. In contrast to closed models, fibril numbers and orientations are this time variables and not given inputs. In this context, two types of cell wall synthesis have been distinguished: diffusive growth and tip growth.

The most common mode of plant cell growth, diffusive growth, involves a homogeneous deposition of new components all over the wall's inner surface. Considering the viscous nature of the cell wall and the addition of new material as an incoming flux, Dyson \& Jensen (2010) modeled the diffusely growing cell wall within the framework of fluid mechanics. The pectin matrix was represented as a viscous fluid and CMFs as rodlike embedded particles. Whereas the previously mentioned models relied only on the equilibrium of forces, here conservation of mass was also included. This implied that the increase in the number of wall components was exactly accounted for by the flux of incoming and outgoing elements at the interface with the cytoplasm. The mechanical behavior of pectins and CMFs was described through equations expressing the strain rate (i.e., relative deformation rate) as a function of the stress field. The general and precise framework of fiber-reinforced fluid dynamics is mathematically very elaborate. To simplify otherwise too-complex equations, assumptions were made on the biological system, which mainly concerned the geometrical descriptions of the cell-basically considered here as a cylinder-and cell wall structure. Using this general framework, Dyson and coworkers (2010) explored the consequences of microfibril orientation and the extensibility perpendicular to the main axis of growth. They explicitly showed how the orientation of microfibrils could impact growth dynamics by establishing a direct mathematical relationship between growth rate and the mean orientation angle of microfibrils. As expected, the more microfibrils are perpendicular to the main cell axis, the easier it is for the cell wall to expand in that direction.

Like in Lockhart's equation, Dyson \& Jensen (2010) found that growth had to be under the control of turgor pressure. However, the yielding threshold, a key element of the Lockhart equation, failed to emerge within this model. They hypothesized that this was because the kinetics of cross-linking was not taken into account. This issue was further investigated in a follow-up study (Dyson et al. 2012), where cross-linking could be regulated by enzymatic activity and mechanical stress (Pien et al. 2001). The system showed a threshold-like behavior (see Figure 3); in other words, different linear relationships between stress and strain rate at respectively small and high stresses were found, but contrary to a pure threshold behavior, where transition between the two regimes occurred abruptly at one value (i.e., the threshold), the transition was spread out over an interval. This smooth transition is a consequence of the cross-linking kinetics, which leads to an exponential relationship between the stress and the strain rate.

In tip-growing cells, such as pollen tubes or root hairs, newly formed components are deposited by a vesicle-based mechanism at the cell's tip only (cf. Gierz \& Bartnicki-Garcia 2001). Cells undergoing tip growth keep the same shape over time, which is simply translated along the growth axis (Goriely \& Tabor 2003). Several models (Dumais et al. 2004, Goriely \& Tabor 2003) show how such maintenance of shape during growth could rely on strong gradients of mechanical wall characteristics. These models are based on the obvious idea that, to yield at the tip only, the wall must be softer at the tip than on the flanks. Taking explicitly into account the weakening of already existing bonds within the pectin matrix by newly deposited pectin monomers, realistic models of tip-growing cells were obtained that explained certain observed features, such as oscillatory
Stress: given a surface of area $S$ and a force $F$ applied on it, the corresponding stress $\sigma$ is defined by $\sigma=F / S$

Field: a physical quantity (number, vector, or matrix) defined at each point of the studied space at any given time 


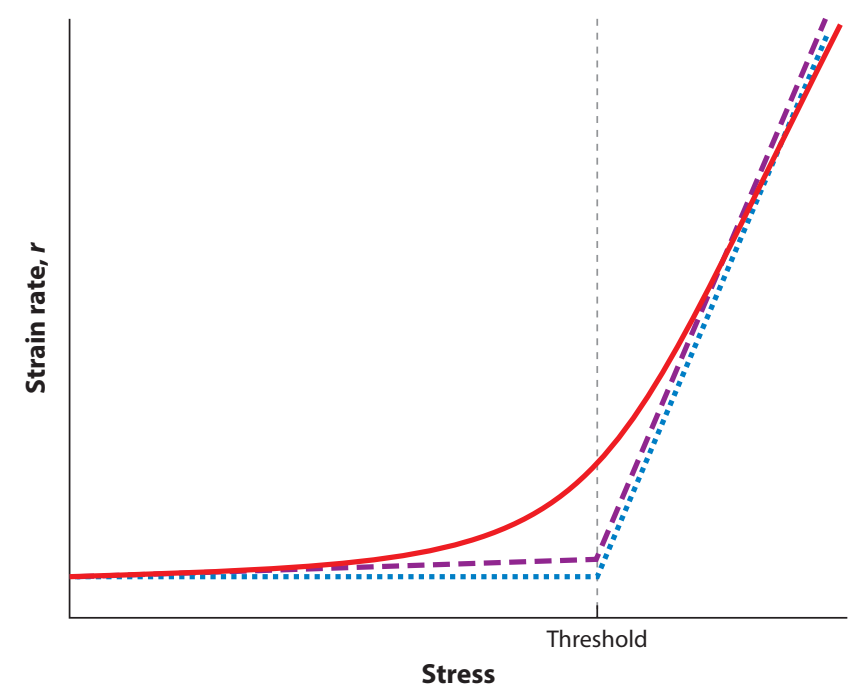

Figure 3

Relationship between stress and strain rate, $r$, adapted from Dyson et al. (2012). The solid red curve represents the growth law as calculated by Dyson et al.; the dashed purple curve illustrates a linear piecewise approximation, also proposed by Dyson et al.; and the dotted blue curve illustrates the growth law, as idealized by Lockhart.

behavior (Rojas et al. 2011). The model showed that in fast-growing cells, incorporation of new components in the wall can be faster than their vesicle-based delivery at the inner wall surface. The resulting shortage of new components triggers a reduction in growth rate, which in turn enables stocks to pile up again, leading, for example, to an acceleration of expansion.

An interesting output of the models of tip-growing cells is that they require the deposition of cellulose fibers in successive layers parallel to the cell membrane. However, a specific alignment of CMFs within these layers is not fundamental, contrary to the models of diffusely growing cells. Thus, in both models structural anisotropy emerges as a fundamental property: In tip-growing cells, this concerns the layered organization of the wall, whereas in diffusely growing cells, the direction of the microfibrils within layers is important (Figure 4).

In conclusion, by taking into account biochemical mechanisms, such as the deposition of newly formed material and the dynamic enzymatic regulation of cross-links, long timescales are explored that cannot be reached by closed system models. Lockhart's equation appears again as a simple but fundamental underlying law governing the growth process. Considering the wall structure, these models show how the original 1D Lockhart model can be extended to 3D, where growth rates in particular directions are regulated by two anisotropic quantities (i.e., the turgor-induced stress pattern within the plane of the cell wall and the mechanical properties of its constituting polymer networks).

In their most recent and sophisticated versions, these physical models of the growing cell rely on fluid mechanics or viscoplasticity theory. Despite their different mathematical formulations, both approaches are part of continuum mechanics and therefore are consistent with each other. Because the concept of a precise yielding threshold is inherent to viscoplasticity (Dumais et al. 2006), related models seem appropriate to study the triggering and initiation of growth. The same concept does not appear naturally in a purely viscous description, making fluid mechanics-based models less efficient to study growth initiation. Conversely, its finer mathematical implementation enables a more precise description of growth itself. 


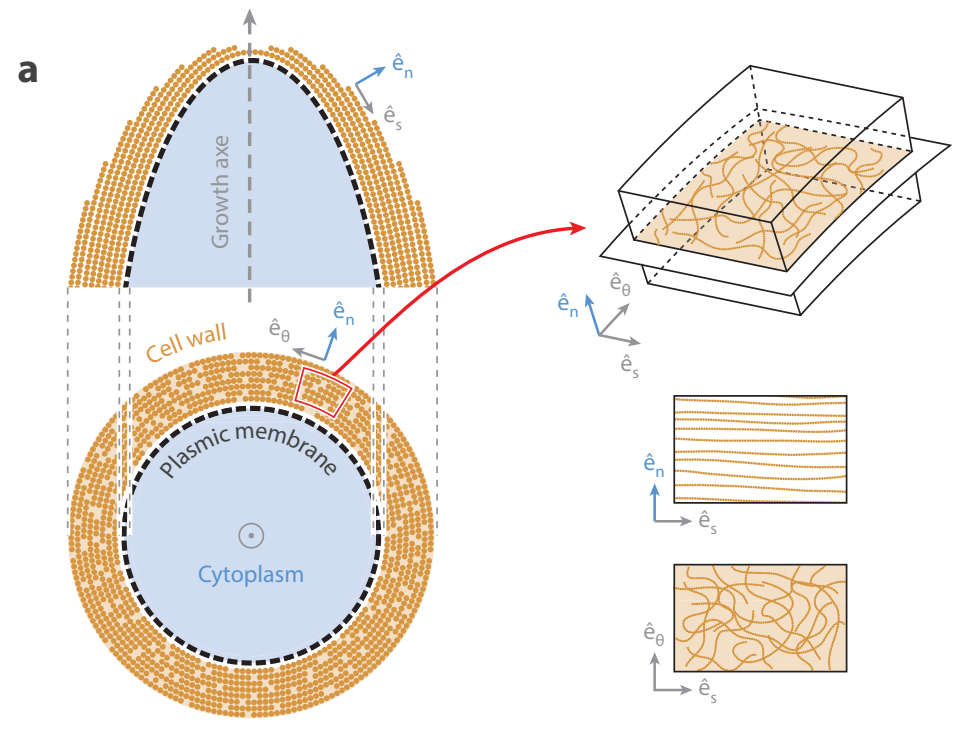

b

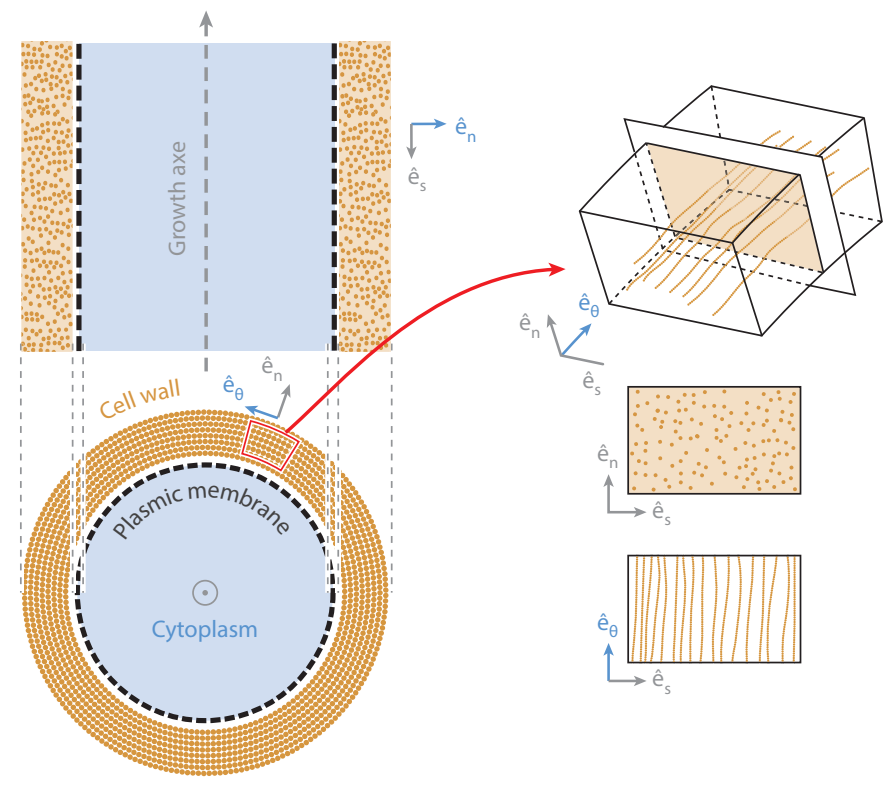

Figure 4

Sketches of the cell wall structure $(a)$ in tip-growing models and $(b)$ in diffusely growing models. Adapted from Dumais et al. (2006). The main direction of anistropy is displayed by a blue vector.

Note that novel directions are being explored. In a recent model, Barbacci et al. (2013) proposed a thermodynamic framework of plant cell growth centered on energy transfers. Growth is described as an interplay between three forms of energy: mechanical, thermal, and chemical. For instance, polymerization of the wall matrix is seen as the transduction of chemical into mechanical energy, and enzymatic activity is seen as a bidirectional coupling between chemical and thermal energies. 
Symplastic growth: growth of a group of cells in which cells do not move with respect to each other

In that framework, Lockhart's and Ortega's equations naturally appear as restricted and simplified versions of a more general, bio-chemico-mechanical theory of plant cell growth.

\section{PHYSICAL MODELS OF TISSUE GROWTH}

How are the basic physical processes at work at the level of individual cells translated into growing tissues and organs? Models of tissue development have appeared only relatively recently in the study of plant morphogenesis. This is at least partially because describing tissue growth takes modeling to another level of complexity, largely because of the highly heterogeneous nature of tissues. First, certain regions may express different combinations of molecular regulators, which may cause them to grow more quickly or slowly than neighboring regions. Second, owing to the symplastic growth of plant tissues, such differences in growth rates generate forces that are readily transmitted throughout the tissue, leading to the accumulation of stresses. Third, the system may privilege certain directions of growth in certain regions or at the level of the entire organ, involving a more or less marked regulation of growth anisotropy. Finally, evidence for interactions between mechanical stresses and genetic regulation (Boudaoud 2010, Hamant et al. 2008, Uyttewaal et al. 2012) further complicates the picture.

In the following, we review the different attempts that have been made to deal with these issues. Starting with deterministic models that regulate growth through the use of mechanics as a trading system between regions, we then consider models in which mechanical stresses feed back on gene regulation. Finally, we discuss recent attempts to assess the robustness of tissue development via the introduction of stochastic components in the growth models.

\section{A Conceptual Framework for Modeling Tissue Growth}

In a seminal paper, Coen and coworkers (2004), based on earlier work from Avery (1933) and Erickson $(1966,1976)$, remarked that, if considered at a sufficiently small scale, every local shape change in a developing organ can be mathematically decomposed into a reduced set of regional transformations corresponding to $(a)$ isotropic dilatation or local growth rate and two parameters linked to growth anisotropy, namely $(b)$ the degree to which a region grows in a particular direction and $(c)$ the control of specific growth directions (see Figure 5). By regulating these parameters independently during growth, genes would thus achieve a variety of different forms.

This conceptual framework provided a mechanistic bridge between molecular regulation and form by mapping infinitesimal molecular information to infinitesimal kinematic deformations.

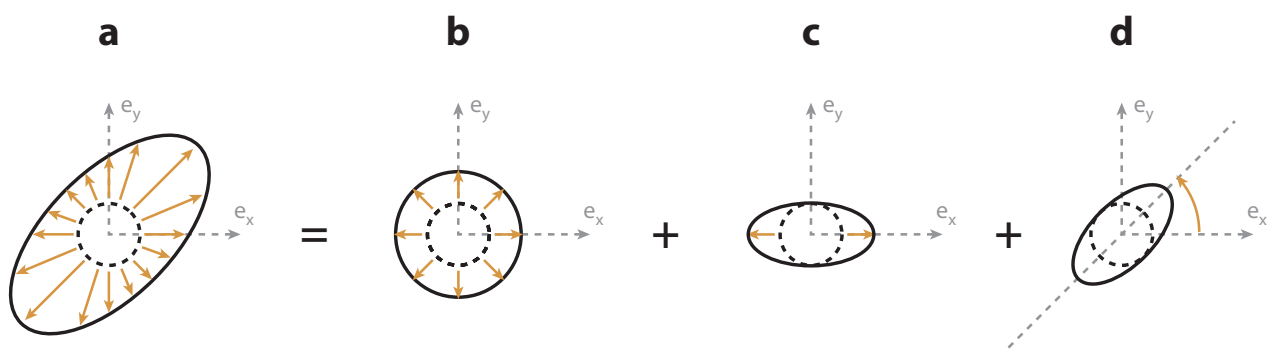

Figure 5

Decomposition of a 2D (or 3D) infinitesimal deformation $(a)$ as the sum of three basis operations: (b) isotropic deformation, $(c)$ ratio of anisotropy, and $(d)$ orientation of the direction of the maximal deformation. For further details, see Coen et al. (2004). 


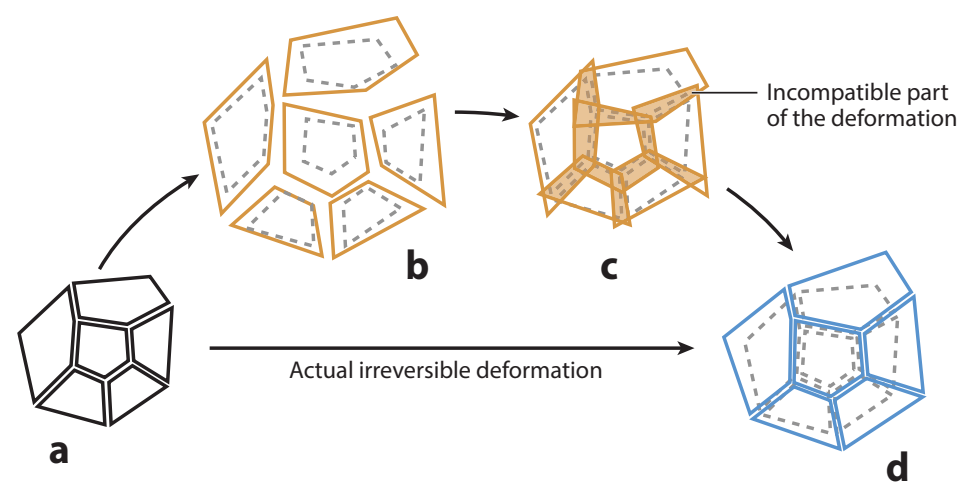

Figure 6

The growing polarized tissue (GPT) framework adapted from Kennaway et al. (2011). (a) Initial state and (b) specified growth each region would reach if isolated. (c) These specified growths are in general incompatible in the actual tissue. $(d)$ Regions realize a physical tradeoff that results in an actual growth shape.

This approach was developed as a full computational system for modeling tissue growth, called the growing polarized tissue (GPT) framework (Green et al. 2010, Kennaway et al. 2011). GPT treats the tissue as a continuum, with information on embedding rate and polarity at each point. The polarity is established by signals that propagate through the tissue from signal sources to signal sinks, both under genetic control. At each point, gene regulatory networks can interact with the local polarity and specify growth rates in directions parallel or perpendicular to it. In this way, genes locally specify growth. However, owing to the competition for space with the other growing parts of the tissue, the specified growth cannot in general be achieved, and an actual growth results from a physical tradeoff between the growing regions, mediated via mechanics. This is the resultant growth (see Figure 6).

The GPT system captures anisotropic growth of tissues, represented as deformable shells, in three dimensions without taking into account individual cells. Mechanical equilibrium between regions is numerically computed using elasticity theory and a computational model where the tissue continuum has been meshed into small polyhedral elements. At each time step, starting from the reference configuration computed at the previous time step, a specified growth is assessed for each region from the local growth rates and anisotropy information, and the resulting growth is then computed. After each time step, the remaining elastic energy is cancelled out, thus resetting the reference configuration of the system to the new computed structure. By regulating regional parameters independently during growth, the system can thus achieve a variety of different forms (Kennaway et al. 2011). The GPT framework was used to propose and test hypotheses for petal formation in Antirrbinum and petal and leaf morphogenesis in Arabidopsis (Green et al. 2010, Kierzkowski et al. 2012, Kuchen et al. 2012, Sauret-Güeto et al. 2013, Schopfer 2006). Importantly, using the framework, the authors were able to evaluate the effect of mutations in several regulatory genes, which led them to propose specific roles for these regulators during morphogenesis.

This work again underlined the importance of mechanics as a key component linking gene regulation to morphogenesis. The notion of region is more general than the concept of the cell and is rather an area of coherent growth and mechanical behavior. Because cells do not appear explicitly in the model, the link between tissue and cell description is left elusive, and gene functions are expressed through abstract terms, such as polarizer or specified growth, or growth rate. Mechanics is used as a tool to enforce tissue integrity but is not considered to reflect precisely real physical forces in tissues. 


\section{Deterministic Models of Multicellular Tissues}

To explore the mechanical link between genes and shape in all its dimensions, new questions must be addressed. What are the biological or physical notions behind polarizer or specified growth introduced in the GPT model? More particularly, how does this relate to the concepts described by Lockhart and others? The problem is complex, combining processes at different spatial (cell and organ) and temporal (chemical reactions and mechanics) scales, from different origins (biological, physical), and with different types of variability (linked to the biological or the measurement processes). In the past decade, several groups started to address these questions and built biophysical models of tissue growth incorporating turgor pressure and more precise descriptions of cell wall remodeling.

Just as for isolated cells, the growth of multicellular tissues can be defined as the irreversible elongation of their cell walls yielding to forces induced by turgor pressure. At first glance, this description of tissue growth would be like a scaled version of single cell models. However, one fundamental difference is linked to the mechanical interactions between cells: Whereas single cells subject to inner pressure can grow freely in space, in a tissue context, cells must deal with the constraints imposed by their neighbors. This idea echoes the notions of specified and resultant growth exposed in the GPT framework. Specified growth corresponds to the growth a single, isolated cell would undergo, and resultant growth corresponds to the actual growth displayed in its multicellular context (Chopard \& Godin 2010; see Figure 6). In other words, in a tissue, cells grow as a consequence of both cell-autonomous and non-cell-autonomous forces (Green et al. 2010; F. Boudon, J. Chopard, O. Ali, O. Hamant, A. Boudaoud, et al., manuscript submitted). To capture this phenomenon, models generally assume that growth rate in a given wall is proportional to the mechanical stress within this wall as soon as it has reached a given threshold value. To compute the growth rate field (i.e., the speed of growth at each point of the tissue and at each time) in a tissue, one must first establish the global stress field within this tissue. The tissue being at each moment at mechanical equilibrium, stresses can be derived from the minimization of the elastic potential energy (see sidebar, Mechanical Energy and Mechanical Equilibrium). Because

\section{MECHANICAL ENERGY AND MECHANICAL EQUILIBRIUM}

In physics, the mechanical equilibrium of a system is achieved when its mechanical energy $U$ is minimal. In the case of $U$ is a function of the position/orientation $p$ and shape $s$ of every cell. Minimizing the system's enlever le "in mes down to finding the position/orientation $p^{*}$ and shape $s^{*}$ of all cells that yield U's mi Physics"

$F_{\text {turgor }}+F_{\text {wall }}=0 \Leftrightarrow U\left(p^{*}, s^{*}\right)=\min _{p, s}\{U(p, s)\}$.
Bmplastic, the mechanical energy of a tissue can be expressed as the sum of the mechanica constituting cell:

$$
U(p, s)=\sum_{i \in c e l l s}\left\{U_{i}\left(p_{i}, s_{i}\right)-P_{i} V_{i}\right\} .
$$

The expression in brackets represents the mechanical energy of the $i^{\text {th }}$ cell. For each cell, the total mechanical energy (also sometimes called the enthalpy) is the sum of a wall-related, usually elastic, energy $U_{i}(p, s)$ and a turgorrelated $\left(-P_{i} V_{i}\right)$ contribution, where $P_{i}$ represents the cell's inner pressure and $V_{i}$ its volume. Although $U_{i}(p, s)$ mathematical expression differs from model to model—according to their geometrical, topological, and mechanical specificities-Equation 6 underlines their common general features. 
of the complexity of multicellular, turgid structures, minimization of the elastic energy and the resulting growth pattern can be calculated only through numerical computation.

To compute mechanically induced growth of a plant tissue with cellular resolution, three main elements must be defined:

- a cellular structure of the tissue, which describes the cells' topology (i.e., their arrangement with respect to each other) and geometry (i.e., their shapes). Note that the initial tissue structure can be constructed in a realistic way by digitizing microscope images in 2D (Baskin 2005, Dupuy et al. 2010, Fozard et al. 2013) or 3D (F. Boudon, J. Chopard, O. Ali, O. Hamant, A. Boudaoud, et al., manuscript submitted).

- a constitutive law of growth, which in mechanosensitive models is usually a generalization of Lockhart's theory for multicellular structures linking growth rate to stress field within the cells' walls.

- a constitutive law of elasticity, which describes how stresses and strains are related in the walls. It is usually expressed by choosing a particular form for the elastic potential energy (see sidebar, Mechanical Energy and Mechanical Equilibrium) (3).

These three core components can be found in every model discussed hereafter.

To run the simulation, boundary conditions that specify geometrical and physical constraints applied on the tissue must be defined (e.g., the base of a meristem dome is kept fixed during the simulation, or tissue is put under tension by external forces). Time is discretized, and the mechanical state of the tissue is iteratively computed from the initial configuration. At each time step, the mechanical equilibrium results from the balance between the turgid forces embedded in the system and the mechanical resistance or yielding to these forces. Because the structure of the tissue can contain many components, finding such a global mechanical balance requires adequate computational methods (see sidebar, The Importance of Computational Tools at the Tissue Scale).

Based on these notions, two modeling approaches can be distinguished (Shapiro et al. 2012):

- cell-centered approaches, in which cells are assimilated to mass points connected with each other by $1 \mathrm{D}$ mechanical elements (e.g., springs) that abstract the overall mechanical interaction between cells, and

- vertex-centered approaches, in which walls are explicitly modeled as mechanical elements (e.g., 1D or 2D springs or rods) connecting cell vertices in two or three dimensions.

\section{THE IMPORTANCE OF COMPUTATIONAL TOOLS AT THE TISSUE SCALE}

One feature common to all tissue-centered models is the central role of numerical simulations. The complexity of multicellular structures imposes the use of computational tools mostly to estimate and display solutions of large numbers of equations that are otherwise unsolvable. In the general field of continuum mechanics, two main techniques are usually adopted: the finite difference and the finite elements methods (FDM and FEM, respectively). In FDM, the continuum is approximated by a finite number of discretization points (e.g., the vertices between adjacent cells). These points are assumed to physically interact, like punctual masses connected by springs. Stresses and displacements in the structure are computed only at the discretization points. In FEM, the continuum (e.g., a 2D surface) is tiled by a finite number of elementary components (e.g., triangles or squares). Mechanical properties are attached to each elementary component. Similarly to FDM, at each time step, the method estimates stresses and displacements only at specific points for each component. However, the method extrapolates these results over all the points of the continuum and makes use of these continuous fields to derive the forces at subsequent time steps. In general, the FEM has better convergence properties than the FDM but is more challenging to implement. 


\section{ELASTICITY, VISCOSITY, AND PLASTICITY}

When loaded with mechanical constraints, real-life materials can exhibit complex deformation behaviors, usually expressed as a combination of three simple ones: elastic, viscous, and plastic behaviors.

An elastic deformation is a reversible one: If the applied load is removed, the material will return to its original shape. Often elastic deformations are supposed linear: deformation $(\epsilon)$ and loading stress $(\sigma)$ are related through a linear relationship, Hooke's law, $\sigma=E \varepsilon$, where $E$ represents the rigidity of the material. Graphically, this behavior is represented by a spring (see Figure 2).

During a viscous deformation, the deformation rate is proportional to the loading stress: $d \varepsilon / d t=m \sigma$, where $m$ represents the extensibility of the material. Viscous deformations are irreversible. When the loading removed, deformation stops but does not fade back to zero; in other words, the system does not come back initial state. Graphically, this behavior is represented by a dashpot (see Figure 2).

Often, materials feature a loading threshold: Deformations are reversible under it and become irreversibl This switching behavior is called plasticity. To that extent, Lockhart's model is a viscoplastic description of the cell grows proportionally to its loading stress only when the latter is above a given threshold.

Depending on the context, different constitutive laws can be hypothesized for the mechanical elements (e.g., elastic, viscoelastic, or viscoplastic; see sidebar, Elasticity, Viscosity, and Plasticity). In 1D, these properties are represented as scalar numbers attached to each part of the structure, including elastic modulus, viscosity, and plastic threshold. In 2D or 3D, these coefficients become arrays of scalars defining the mechanical properties in the different directions of space (structural anisotropy of material) and their coupling.

The first model based on a mechanical interaction between cells was a cell-centered model developed for the growth of a meristematic dome in 3D (Hamant \& Traas 2009, Jönsson et al. 2006). This first abstraction of mechanical interactions between cells was able to maintain the dome shape of the meristem in time. However, for the sake of computational efficiency, the cells were allowed to slide along each other, which is never observed in vivo. To achieve a good trade-off between mechanical realism and computational efficiency, most of the models have subsequently been developed as vertex-centered models in the plane. Dupuy et al. $(2006,2008)$ developed a generic model of tissue morphogenesis that explicitly expresses the physical interactions between cells using a viscous model. Tissues are modeled as planar structures, where cells are represented as polygons in the plane and walls as the edges between the polygon vertices. These edges are associated with $1 \mathrm{D}$ beam elements that can be stretched and bent by external loads. To relate turgor pressure to cell wall expansion, a viscous model at mechanical equilibrium was used, where the strain rate of the beams is directly proportional to the turgor-induced stresses in the walls. With this model, Dupuy et al. (2008) analyzed the distribution of stresses and strains during the emergence of a primordium at the shoot apical meristem by coupling predicted auxin -an important growth-related hormone in plants-distributions and fluxes to an empirical relation between wall viscosity and cellular hormone concentrations. They also applied their approach to examine self-organizing properties of the development of Coleocheate scutata, a microscopic freshwater alga (Dupuy et al.2010). Similar 2D models were proposed with variations on the constitutive laws. Corson et al. (2009), for instance, used a viscoelastic approach to study plant meristems that were no longer able to control growth anisotropy after biochemical treatment. Merks et al. (2011) developed a generic computational framework to simulate the growth of 2D multicellular tissues. Based on a 2D and multicellular version of Dyson et al.'s cellular model, Fozard et al. (2013) used a viscous model to explore the growth and bending of root axis development. 


\section{CELL DIVISION MODELS}

The growth of most plant tissues is accompanied by processes of cell division. The determination of new cell wall timing and positioning during cell division in plants has been largely discussed in the literature since the nineteenth century. Most of the rules assume that cells divide when their volume exceeds a critical size. However, several rules have been conjectured and are still discussed concerning the orientation of the new walls. The most widely spread rule was postulated by Errera (1888) by analogy with soap bubbles. This rule stipulates that the new wall should be formed in a way that minimizes the area of the new wall and splits the mother cell into two equal volumes. Besson \& Dumais (2011) recently analyzed and interpreted this empirical rule in terms of its mechanistic foundation; they could derive a stochastic version of the rule based on the distribution of distances to the cell nucleus. Other division rules are also frequently used in the literature. Hofmeister's rule, for instance, takes into account the dynamics of cell growth and predicts that the new wall will form perpendicular to the main axis of elongation (Hofmeister 1863). Sachs's rule states that the new wall must meet the parent cell's walls at right angles (Sachs 1878).

The models discussed above did not explicitly take into account elasticity as a separate parameter. To address this limitation, elastoplastic models have been developed. Instead of expressing growth directly in terms of elongation owing to a viscous force, growth is modeled as the change in the rest length of the elastic elements as soon as the deformation reaches a given threshold. Hamant et al. (2008), for example, proposed such a model to study the mechanical regulation of growth of the outer layer of the shoot apical meristem. They represented the dome structure of the meristem epidermis by a surface made up of $2 \mathrm{D}$ polygonal cells in $3 \mathrm{D}$ space. Cell walls were explicitly assumed to be elastic and represented as springs. The cellular nature of the inner layers was not represented, and their action on the epidermis was abstracted as a uniform pressure from below the surface. Once mechanical equilibrium was reached, the elastic strain of each wall was compared with a plastic threshold value. When the length was above this value, the rest length of the wall was augmented proportionally to the difference between the strain and the plastic threshold.

In all these models, cell division has been integrated to accompany cell growth. Growth induces the enlargement of cells that divide as soon as they reach some critical size. Different criteria have been used to place the new walls (see sidebar, Cell Division Models) (4). From a modeling point of view, cell division can be seen as a means for remeshing the tissue as it grows. However, most of the previously discussed models reported that cell division adds mechanical elements but has little to no impact on the overall tissue behavior.

\section{Integrating Mechanical Feedback in Multicellular Tissues}

Several studies investigated the possible feedback of mechanical forces on cellular regulation (Hamant et al. 2008; Heisler et al. 2007, 2010). Experimental evidence coming from this work indicated that in certain growing tissues, in particular the shoot apical meristem, cells deposit new CMFs along the main stress axis. This process, which stiffens cells along their maximal stress direction, involves a feedback loop, where mechanical constraints induce specific arrangements of the cortical microtubule-a key player in the microfibril-deposition mechanism (see above; Dyson \& Jensen 2010) - in turn influencing the deposition of CMFs and the cell wall structural anisotropy. A model was used to study the properties and implications of such a mechanical feedback at the scale of the whole meristem (Hamant et al. 2008). This was achieved by tuning elasticity according to the wall's orientation in the overall stress field: Walls oriented in the 
main stress direction were made stiffer than walls that were not. Simulations showed that this mechanism was able to reproduce specific morphogenetic events, such as the formation of a meristematic dome or a cylindrical stem and tissue folding during organ boundary formation. The model was also able to reproduce cortical microtubule reorientation during cell ablation experiments. Modeling combined with experimentation was thus used to support the hypothesis that mechanics acts as both a triggering signal and a steering force in defining growth directions. In a follow-up study, Heisler et al. (2010) also showed that a mechanism that aligns transport of the plant hormone auxin to force fields has patterning properties. Auxin is transported from cell to cell by transmembrane transporter proteins. These transporters are often localized on one side of the cell only. This directed transport creates auxin maxima and auxin minima in tissues, which are subsequently interpreted in terms of differential growth and gene expression. This process constitutes the basis of organ initiation at the shoot apical meristem (Pien et al. 2001, Reinhardt et al. 2003). Experimental evidence indicated that applying external mechanical constraints to the meristem could influence the localization of auxin transporters. A model was proposed in which these proteins are sent by the cell to its most stretched membrane. Simulations showed that such a simple scenario could generate transporter distributions similar to the ones observed in vivo.

Although experimental evidence supported a stress-based reinforcement of the wall, at least part of the results could also be explained by an alternative hypothesis, in which cells align their CMFs perpendicular to the direction of maximal strain. In a curved, anisotropic material, such as the meristem's outer cell wall, this strain-based feedback mechanism is expected to give results similar to those of the stress-based mechanism. In a recent article, Bozorg et al. (2014) went beyond intuition and studied both feedback mechanisms in detail. They set up a precise mechanical model of the meristematic outer cell wall, described as a $2 \mathrm{D}$ shell in $3 \mathrm{D}$ space. Both the degree and direction of its maximum rigidity can be locally tuned by mechanics-based feedback loops. By comparing outputs of simulations between strain-based and stress-based loops, they concluded that the stress-based mechanism reproduced and explained all observed patterns of structural anisotropy, whereas the strain-based one could not.

\section{Toward the Integration of Stochasticity in Models of Multicellular Tissues}

The different models reviewed up to now are deterministic. However, biological systems often contain intrinsic variability that cannot be captured by deterministic rules. To account for such phenomena, it is necessary to include stochasticity.

Uyttewall et al. (2012) recently developed a stochastic approach to study tissue growth, investigating the influence of variability in the individual growth rate of cells on the global growth of a tissue. To do so, they generated a 2D simulated tissue. This tissue is modeled as a flat, polygonal tiling of space with isotropic mechanical properties. Cells and walls are respectively represented by the tiles and their edges. Each cell is considered to have a target growth rate (TGR), which is the growth rate the cell would display if it were isolated, reminiscent of the notion of specified growth introduced above. Based on the cell's intrinsic variability, this TGR is supposed to show random fluctuations across the tissue. Because plant cells in a tissue are not allowed to move relative to each other, two neighboring cells displaying different TGRs would generate mechanical stress between them. To test the possible effects of a mechanical feedback, the authors postulated a reduced TGR in the direction of the maximal stress. This creates a feedback loop between growth rate variability and mechanical stress: Growth variability induces stresses that in turn modify local growth rates.

The influence of two key parameters, namely the fluctuation degree of the TGR (how different individual TGRs can be) and the strength of the feedback loop (how much the TGR is modified 
by stress), on the variability of tissue growth was analyzed. Growth variability within the tissue was quantified through a comparison of growth between a cell and its nearest neighbors. In homogeneously growing tissues, all cells grow at the same rate, and variability is close to zero; conversely, at the boundary between two regions growing at different rates, growth variability is high.

In tissues where the TGR varies significantly from cell to cell, a mild mechanical-based feedback mechanism tends to reduce effective growth-rate differences between cells. Conversely, a strong feedback tends to enhance those differences. By contrast, in homogeneous tissues where TGR fluctuations are low, effective growth variability mainly increases as feedback strength increases. This suggests that, in homogeneous tissues, mechanical feedback promotes local variations of effective growth rate. This could be particularly important in organ outgrowth.

In parallel to this theoretical analysis, the authors investigated the biological mechanism behind the mechanical feedback. In this context, the role of the katanin protein, a protein involved in microtubule dynamics and required for the formation of ordered microtubule arrays (Wasteneys \& Ambrose 2009), was analyzed (Uyttewaal et al. 2012). Experiments showed that katanin-deficient mutants feature ill-formed organs and poorly defined organ boundaries. Overall, the experimental work suggested that katanin, via its effect on microtubule dynamics, promotes local growth-rate variability. This, in turn, leads to growth heterogeneity within the tissue, thus favoring a clear distinction between an emerging organ and the rest of the meristematic tissue.

This feedback from mechanics on the regulation of growth opened up new perspectives for analyzing the role of cell division during growth. Alim et al. (2012) investigated the influence of cell division orientation on growth heterogeneity. Five possible hypotheses were investigated. Interestingly, scenarios where cells divided in random orientations generated heterogeneously growing tissues displaying anisotropic growth. New cross walls following the shortest path between existing walls or division planes along the direction of maximal mechanical stress reduced growth heterogeneity by enhancing the regulation of growth by mechanical stresses. Moreover, the model could quantitatively confirm the qualitative idea that cell division is a global stress-releasing mechanism.

\section{CONCLUSIONS}

To control shape changes during development, molecular networks must control local growth rates and directions. In this review, we underlined the importance of physical processes in this context. As we have seen, it is widely accepted that turgor (as a driving force) and the cell wall (as the most prominent mechanical component) play central roles in morphogenesis. In this context, the equation proposed by Lockhart, describing growth as an irreversible yielding of the wall to turgor, stands out as the basic principle in many of the models discussed above. The impressive advances made over the past decades have started to provide an understanding of how this principle arises from the molecular control of cell wall mechanics and local osmotic pressure. Studies at the level of entire tissues have further completed this picture, pointing to the importance of physical interactions between cells and the constraints generated by the collective behavior of multiple cells.

With this increasing knowledge, models taking into account the physical and molecular/genetic basis of growth have become essential tools for our understanding of morphogenesis. Modeling approaches have helped to define and formalize complex hypotheses on how plant growth is regulated at multiple scales. This increased use of modeling has also had a profound impact on the way growing organisms are studied. In particular, to construct, test, and further refine the models, quantitative information is required on a wide variety of parameters, from chemical concentrations to mechanical properties and local growth characteristics (Burgert 2006, Dumais \& Kwiatkowska 
2002, Fernandez et al. 2010, Grandjean et al. 2004, Heisler et al. 2007, Reddy et al. 2004). Although novel, quantitative imaging methods have been developed and the measurement of cell mechanics has come within reach, obtaining this information remains one of the main challenges for the near future.

Beyond biology and physics, the generation of physical models in the form of virtual tissues also requires novel developments in the field of computer science. The use of always faster computers helps implement increasingly complex models, but this is not sufficient. Existing software must be further optimized; in particular, we must develop 3D-finite-elements-based simulations of whole tissues with mechanical elements able to capture anisotropic properties in multiple directions.

\section{DISCLOSURE STATEMENT}

The authors are not aware of any affiliations, memberships, funding, or financial holdings that might be perceived as affecting the objectivity of this review.

\section{ACKNOWLEDGMENTS}

Olivier Ali and Jan Traas are funded by an ERC fellowship ("Morphodynamics"), and Christophe Godin and Jan Traas are supported by an INRIA grant ("Morphogenetics").

\section{LITERATURE CITED}

Alim K, Hamant O, Boudaoud A. 2012. Regulatory role of cell division rules on tissue growth heterogeneity. Front. Plant Sci. 3:174

Avery GS Jr. 1933. Structure and development of the tobacco leaf. Am. F. Bot. 20:565-92

Barbacci A, Lahaye M, Magnenet V. 2013. Another brick in the cell wall: biosynthesis dependent growth model. PLOS ONE 8(9):e74400

Baskin T. 2005. Anisotropic expansion of the plant cell wall. Annu. Rev. Cell Dev. Biol. 21:203-22

Besson S, Dumais J. 2011. Universal rule for the symmetric division of plant cells. Proc. Natl. Acad. Sci. USA 108(15):6294-99

Boudaoud A. 2010. An introduction to the mechanics of morphogenesis for plant biologists. Trends Plant Sci. 15(6):353-60

Bozorg B, Krupinski P, Jönsson H. 2014. Stress and strain provide positional and directional cues in development. PLOS Comput. Biol. 10(1):e1003410

Burgert I. 2006. Exploring the micromechanical design of plant cell walls. Am. F. Bot. 93(10):1391-401

Cho H-T, Cosgrove DJ. 2000. Altered expression of expansin modulates leaf growth and pedicel abscission in Arabidopsis thaliana. Proc. Natl. Acad. Sci. USA 97(17):9783-88

Chopard J, Godin C. 2010. The role of mechanics in morphogenesis. Proc. 6th Int. Workshop Funct.-Struct. Plant Models, Davis, CA, pp. 135-37. Davis: Regents Univ. Calif.

Coen E, Rolland-Lagan AG, Matthews M, Bangham JA, Prusinkiewicz P. 2004. The genetics of geometry. Proc. Natl. Acad. Sci. USA 101(14):4728-35

Corson F, Hamant O, Bohn S, Traas J, Boudaoud A, Couder Y. 2009. Turning a plant tissue into a living cell froth through isotropic growth. Proc. Natl. Acad. Sci. USA 106(21):8453-58

Cosgrove DJ. 1993. Wall extensibility: its nature, measurement and relationship to plant cell growth. New Phytol. 124(1):1-23

Cosgrove DJ. 2005. Growth of the plant cell wall. Nat. Rev. Mol. Cell Biol. 6(11):850-61

Dumais J, Kwiatkowska D. 2002. Analysis of surface growth in shoot apices. Plant 7. 31(2):229-41

Dumais J, Long SR, Shaw SL. 2004. The mechanics of surface expansion anisotropy in Medicago truncatula root hairs. Plant Physiol. 136(2):3266-75

Dumais J, Shaw SL, Steele CR, Long SR, Ray PM. 2006. An anisotropic-viscoplastic model of plant cell morphogenesis by tip growth. Int. F. Dev. Biol. 50(2-3):209-22 
Dupuy L, Mackenzie J, Haseloff J. 2010. Coordination of plant cell division and expansion in a simple morphogenetic system. Proc. Natl. Acad. Sci. USA 107(6):2711-16

Dupuy L, Mackenzie J, Rudge T, Haseloff J. 2008. A system for modelling cell-cell interactions during plant morphogenesis. Ann. Bot. 101(8):1255-65

Dupuy L, Mackenzie JP, Haseloff JP. 2006. A biomechanical model for the study of plant morphogenesis: Coleocheate orbicularis, a 2D study species. Proc. 5th Plant Biomech. Conf., Stockholm

Dyson RJ, Band LR, Jensen OE. 2012. A model of crosslink kinetics in the expanding plant cell wall: yield stress and enzyme action. F. Theor. Biol. 307:125-36

Dyson RJ, Jensen OE. 2010. A fibre-reinforced fluid model of anisotropic plant cell growth. F. Fluid Mech. 655:472-503

Erickson RO. 1966. Relative elemental rates and anisotropy of growth in area: a computer programme. 7 . Exp. Bot. 17(2):390-403

Erickson RO. 1976. Modeling of plant growth. Annu. Rev. Plant Physiol. 27(1):407-34

Errera L. 1886. Sur une condition fondamentale d'équilibre des cellules vivantes [On a fundamental condition of equilibrium for living cells]. C. R. Hebd. Séances Acad. Sci. 103:822-24

Fernandez R, Das P, Mirabet V, Moscardi E, Traas J, et al. 2010. Imaging plant growth in 4D: robust tissue reconstruction and lineaging at cell resolution. Nat. Meth. 7(7):547-53

Fleming AJ, McQueen-Mason S, Mandel T, Kuhlemeier C. 1997. Induction of leaf primordia by the cell wall protein expansin. Science 276(5317):1415-18

Fozard A, Lucas M, King JR, Jensen OE. 2013. Vertex-element models for anisotropic growth of elongated plant organs. Front. Plant Sci. 4:233

Geitmann A, Ortega JKE. 2009. Mechanics and modeling of plant cell growth. Trends Plant Sci. 14(9):467-78

Gierz G, Bartnicki-Garcia S. 2001. A three-dimensional model of fungal morphogenesis based on the vesicle supply center concept. F. Theor. Biol. 208(2):151-64

Goriely A, Tabor M. 2003. Biomechanical models of hyphal growth in actinomycetes. F. Theor. Biol. 222(2):211-18

Grandjean O, Vernoux T, Laufs P, Belcram K, Mizukami Y, Traas J. 2004. In vivo analysis of cell division, cell growth, and differentiation at the shoot apical meristem in Arabidopsis. Plant Cell 16(1):74-87

Green AA, Kennaway JR, Hanna AI, Bangham JA, Coen E. 2010. Genetic control of organ shape and tissue polarity. PLOS Biol. 8(11):e1000537

Green PB. 1968. Growth physics in Nitella: a method for continuous in vivo analysis of extensibility based on a micro-manometer technique for turgor pressure. Plant Physiol. 43(8):1169-84

Green PB, Erickson RO, Buggy J. 1971. Metabolic and physical control of cell elongation rate: in vivo studies in Nitella. Plant Physiol. 47(3):423-30

Hamant O, Heisler MG, Jonsson H, Krupinski P, Uyttewaal M, et al. 2008. Developmental patterning by mechanical signals in Arabidopsis. Science 322(5908):1650-55

Hamant O, Traas J. 2009. The mechanics behind plant development. New Phytol. 185(2):369-85

Heisler M, Alim K, Jönsson H, Hamant O, Boudaoud A. 2007. Modelling meristem development in plants. Curr. Opin. Plant Biol. 10(1):92-97

Heisler MG, Hamant O, Krupinski P, Uyttewaal M, Ohno C, et al. 2010. Alignment between PIN1 polarity and microtubule orientation in the shoot apical meristem reveals a tight coupling between morphogenesis and auxin transport. PLOS Biol. 8(10):e1000516

Hofmeister W. 1863. Zusätze und Berichtigungen zu den 1851 veröffentlichen Untersuchungen der Entwicklung höherer Kryptogamen [Additions and adjustments to the 1851 published investigations of the development of higher cryptogams]. Fahrb. Wiss. Bot. 3:259-93 (in German)

Jönsson H, Heisler MG, Shapiro BE, Meyerowitz EM, Mjolsness E. 2006. An auxin-driven polarized transport model for phyllotaxis. Proc. Natl. Acad. Sci. USA 103(5):1633-38

Kennaway R, Coen E, Green A, Bangham A. 2011. Generation of diverse biological forms through combinatorial interactions between tissue polarity and growth. PLOS Comput. Biol. 7(6):e1002071

Kha H, Tuble SC, Kalyanasundaram S, Williamson RE. 2010. WallGen, software to construct layered cellulose-hemicellulose networks and predict their small deformation mechanics. Plant Physiol. 152(2):774-86 
Kierzkowski D, Nakayama N, Routier-Kierzkowska A-L, Weber A, Bayer E, et al. 2012. Elastic domains regulate growth and organogenesis in the plant shoot apical meristem. Science 335(6):1096-99

Kuchen EE, Fox S, Barbier de Reuille P, Kennaway R, Bensmihen S, et al. 2012. Generation of leaf shape through early patterns of growth and tissue polarity. Science 335(6072):1092-96

Lecuit T, Lenne P-F. 2007. Cell surface mechanics and the control of cell shape, tissue patterns and morphogenesis. Nat. Rev. Mol. Cell Biol. 8(8):633-44

Lockhart JA. 1965. An analysis of irreversible plant cell elongation. 7. Theor. Biol. 8(2):264-75

Lockhart JA. 1967. Physical nature of irreversible deformation of plant cells. Plant Physiol. 42(11):1545-52

McQueen-Mason S, Durachko DM, Cosgrove DJ. 1992. Two endogenous proteins that induce cell wall extension in plants. Plant Cell Online 4:1425-33

Merks RMH, Guravage M, Inze D, Beemster GTS. 2011. VirtualLeaf: an open-source framework for cellbased modeling of plant tissue growth and development. Plant Physiol. 155(2):656-66

Ortega JK. 1985. Augmented growth equation for cell wall expansion. Plant Physiol. 79(1):318-20

Paredez AR, Somerville CR, Ehrhardt DW. 2006. Visualization of cellulose synthase demonstrates functional association with microtubules. Science 312(5779):1491-95

Passioura JB, Fry SC. 1992. Turgor and cell expansion: beyond the Lockhart equation. Funct. Plant Biol. 19(5):565-76

Peaucelle A, Braybrook S, Höfte H. 2012. Cell wall mechanics and growth control in plants: the role of pectins revisited. Front. Plant Sci. 3:121

Pien S, Wyrzykowska J, McQueen-Mason S, Smart C, Fleming A. 2001. Local expression of expansin induces the entire process of leaf development and modifies leaf shape. Proc. Natl. Acad. Sci. USA 98(20):11812-17

Reddy GV, Heisler MG, Ehrhardt DW, Meyerowitz EM. 2004. Real-time lineage analysis reveals oriented cell divisions associated with morphogenesis at the shoot apex of Arabidopsis thaliana. Development 131(17):4225-37

Reinhardt D, Pesce E-R, Stieger P, Mandel T, Baltensperger K, et al. 2003. Regulation of phyllotaxis by polar auxin transport. Nature 426(6964):255-60

Rojas ER, Hotton S, Dumais J. 2011. Chemically mediated mechanical expansion of the pollen tube cell wall. Biophys. F. 101(8):1844-53

Sachs J. 1878. Über die Anordnung der Zellen in jüngsten Pflanzentheilen [On the arrangement of cells in embryonic tissues]. Arb. Bot. Inst. Wïrzbg. 2:46-104 (in German)

Sauret-Güeto S, Schiessl K, Bangham A, Sablowski R, Coen E. 2013. JAGGED controls Arabidopsis petal growth and shape by interacting with a divergent polarity field. PLOS Biol. 11(4):e1001550

Schopfer P. 2006. Biomechanics of plant growth. Am. F. Bot. 93(10):1415-25

Shapiro BE, Jönsson H, Sahlin P, Heisler M, Roeder A, et al. 2012. Tessellations and pattern formation in plant growth and development. arXiv: 1209:2937

Uyttewaal M, Burian A, Alim K, Landrein B, Borowska-Wykręt D, et al. 2012. Mechanical stress acts via katanin to amplify differences in growth rate between adjacent cells in Arabidopsis. Cell 149(2):439-51

Veytsman BA, Cosgrove DJ. 1998. A model of cell wall expansion based on thermodynamics of polymer networks. Biophys. 7. 75(5):2240-50

Wasteneys GO, Ambrose JC. 2009. Spatial organization of plant cortical microtubules: close encounters of the 2D kind. Trends Cell Biol. 19(2):62-71

Willats WGT, Knox JP, Mikkelsen JD. 2006. Pectin: New insights into an old polymer are starting to gel. Trends Food Sci. Technol. 17(3):97-104

Wolf S, Hématy K, Höfte H. 2012. Growth control and cell wall signaling in plants. Annu. Rev. Plant Biol. 63(1):381-407

Yi H, Puri VM. 2012. Architecture-based multiscale computational modeling of plant cell wall mechanics to examine the hydrogen-bonding hypothesis of the cell wall network structure model. Plant Physiol. 160(3):1281-92 